\title{
Kernos
}

Revue internationale et pluridisciplinaire de religion grecque antique

$17 \mid 2004$

Varia

\section{Angelo BRELICH, Mitologia, politeismo, magia e altri studi di storia delle religioni (1946-1977)}

\section{Francesca Prescendi}

\section{OpenEdition \\ Journals}

\section{Édition électronique}

URL : http://journals.openedition.org/kernos/1515

DOI : 10.4000/kernos. 1515

ISSN : 2034-7871

\section{Éditeur}

Centre international d'étude de la religion grecque antique

Édition imprimée

Date de publication : 1 janvier 2004

ISSN : 0776-3824

\section{Référence électronique}

Francesca Prescendi, « Angelo вRELıCH, Mitologia, politeismo, magia e altri studi di storia delle religioni (1946-1977) », Kernos [En ligne], 17 | 2004, mis en ligne le 22 avril 2011, consulté le 24 septembre 2020. URL : http://journals.openedition.org/kernos/1515 ; DOI : https://doi.org/10.4000/kernos.1515 
s'agit non seulement d'un exposé théologique sous la forme de la littérature traditionnelle, mais aussi de l'expression, allusive mais bien réelle, d'espérances personnelles. Prêtre à Delphes et officiant pour les cultes de la cité de Chéronée, Plutarque exprime ses convictions par rapport à l'importance de la fonction de la communauté dans la pratique religieuse.

La troisième communication (p. 93-116) est consacrée à un état des recherches à propos de l'importance respective des influences phéniciennes et anatoliennes sur la culture grecque. L'A. pose un regard critique sur les différentes interventions de Santo Mazzarino : replaçant les éléments de la problématique dans un large contexte idéologique, il conclut à l'importance des courants d'Asie mineure, notamment indoeuropéens, sur les populations grecques depuis l'époque archaïque mais surtout au rôle prépondérant de l'activité maritime des Grecs depuis le viII" siècle dans le processus de stimulation culturelle.

La quatrième partie (p. 117-150) appréhende la conception de l'être humain intérieur dans les doctrines platonicienne et paulinienne. Encore aujourd'hui, les progrès de la médecine n'ont pas fait disparaître la croyance en l'existence d'une âme à l'intérieur du corps qui constituerait une personne interne distincte de la personne corporelle. La présence de formules de ce genre dans la philosophie platonicienne et dans la tradition chrétienne démontre non seulement la persistence du concept de corps et d'âme, mais également son adaptation aux contextes historico-culturels. Immortelle et manifestée de manière psycho-somatique chez Homère, l'âme chez Platon apparaît non seulement séparable du corps, mais également immatérielle et incorporelle au sens le plus profond, c'est-à-dire inaccessible aux sens et impossible à localiser dans aucune partie, intérieure ou extérieure, du corps. Le concept a été repris et développé par d'autres auteurs : Sénèque, Marc Aurèle, Porphyre. Cette communication est la seule des cinq contributions reprises dans ce volume à ne pas comporter de bibliographique finale récapitulative, mais les notes de bas de page compensent avantageusement cette absence.

La cinquième et dermière partie (p. 151-178) aborde le problème de la violence dans l'histoire religieuse à travers la répression du manichéisme. Malgré la valeur incontestable accordée au langage, force est de reconnaître l'existence d'un espace de violence à l'intérieur des sociétés humaines antiques: la violence est légitimée, notamment dans sa forme pénale. Fondé sur le scandale créé par l'exécution d'un innocent, le message chrétien est articulé paradoxalement sur le rejet de toutes les formes de violence et d'agression, et sur l'annonce d'une paix chantée par une armée céleste et gagnée au prix d'un combat final contre les démons. L'infléchissement de la politique impériale sous le règne de Constantin va normaliser le recours à la violence pénale en faveur du christianisme, notamment dans le cadre de la lutte contre les dissidents religieux, comme les manichéens.

Catherine Lheureux-Godbille (FNRS - Université de Liège)

Angelo BreliCH, Mitologia, politeismo, magia e altri studi di storia delle religioni (1946-1977), a cura di Paolo Xella, Napoli, Liguori Editore, 2002. 1 vol. $16 \times 24 \mathrm{~cm}$, XII +182 p. (Anthropos, 38). ISBN : 88-207-3415-X.

Paolo Xella publie un recueil des travaux de l'historien des religions italien Angelo Brelich (1913-1977), vingt-cinq ans après la mort de celui-ci. Comme il le précise dans la préface, ce livre réunit les articles de Brelich parus dans la revue Studi e materiali di storia delle religioni - dont Brelich était l'éditeur -, ainsi que ceux qui furent présentés 
dans des volumes collectifs. Des notes plus brèves, écrites par Brelich au début de son activité scientifique, mais révélatrices de sa méthode de travail, y trouvent également leur place. Les articles qui ont déjà été republiés (surtout dans A. Brelich, Storia delle religioni perché?, Liguori, Napoli, 1979), sont par contre exclus, même si leur importance est indéniable.

Ceux qui ont déjà eu l'occasion d'être confrontés à l'outure de Brelich peuvent se rendre compte à première vue de l'importance et de l'intérêt pratique de ce volume. Par exemple, le fameux article "Der Polytheismus », publié pour la première fois en 1960 dans Numen, se trouve ici, en traduction italienne, à côté de l'autre étude que Brelich a dédié au même sujet, «Politeismo e soteriologia » et qui fut, quant à elle, publiée dans un mélange en l'honneur de S.G.F. Brandon (1963). Les «tre note (sul concetto di magia)", parues en 1976 dans un recueil édité par le même Paolo Xella, sont réinsérées ici, pour le grand avantage des lecteurs : ce texte constitue en effet une référence incontournable pour tous ceux qui travaillent sur la magie dans la civilisation romaine. Une autre perle est l'article concernant les sacrifices humains «Simbolo di un simbolo ", paru originellement en anglais dans un volume d'hommage à M. Eliade et traduit pour la première fois en italien. Or, à côté de ces études fondamentales, ce volume permet aussi de découvrir certains écrits moins connus, mais révélateurs de la personnalité de Brelich. C'est le cas par exemple de "La religione greca in Sicilia » présenté lors d'un colloque en Sicile. Brelich déclare vouloir parler exclusivement de la religion grecque et non pas de celles de la Sicile pré-grecque. Les raisons quil invoque sont le manque de documents permettant une connaissance en profondeur des religions indigènes et, surtout, l'impossibilité de discerner en quoi ces religions ont influencé celle des colons grecs. Il nie ainsi la possibilité de démontrer la continuité directe entre les cultures originaires de la Sicile et celle des colons grecs, préoccupation qui était chère aux organisateurs du colloque, comme on le comprend au ton polémique de l'article.

Un autre texte qu'on a plaisir à trouver dans ce recueil est le dernier : « La metodologia della scuola di Roma». Il s'agit de la contribution que Brelich a présentée au colloque sur le mythe grec organisé à Urbino en 1973, dont les actes ont été publiés par B. Gentili et G. Paioni (1977). Dans l'introduction de sa conférence, Brelich donne des informations sur l'ambiance intellectuelle qui l'entoure. Il affirme utiliser la dénomination de "scuola romana " pour la première fois dans un contexte public, et se préoccupe de bien expliquer la manière dont il faut la comprendre. La «scuola romana di storia delle religioni », précise Brelich, n'est ni un groupe constitué par un maître et ses disciples, ni un ensemble de collègues qui partagent nécessairement les mêmes intérêts ou des positions théoriques prédéfinies. Il s'agit plutôt d'un cercle d'amis qui, grâce à de fréquents contacts résultants d'un même lieu de travail (l'Istituto di Studi storico-religiosi dell'Università di Roma), ont des échanges d'idées constants. La raison déterminante pour définir ce groupe comme une «scuola » est sa descendance scientifique d'un ancêtre commun: Raffaele Pettazzoni, l'«unico studioso italiano di storia delle religioni ». Dans la suite de l'intervention qui porte sur le mythe en Grèce, Brelich illustre la méthode de son école, à savoir le «comparatisme historique ». Ce qui intéresse la «scuola di Roma », affirme-t-il, est d'étudier non pas un concept de mythe immuable et éternel, mais la production mythologique d'une civilisation tout au long de son histoire. En effet, le mythe est une création humaine qui se modifie dans le temps. Pour ceux qui adoptent la méthode du comparatisme historique ces changements sont fondamentaux, et constituent le centre du problème. A la fin de sa communication, Brelich exprime son désir de se confronter avec les méthodes de travail des autres participants du colloque, telles que le structuralisme et 
l'herméneutique de la psychanalyse, qui permettront, selon son avis, de dégager le problème du mythe du contexte spécifique de la culture grecque pour atteindre des affirmations plus globales, valables pour toute l'humanité.

Malheureusement, Brelich n'a pas pu transmettre sa volonté de collaboration en héritage à la «scuola di Roma ». P. Xella, à la fin de son introduction, affirme que cette école n'existe plus : elle s'est dissoute. Ce livre, poursuit-t-il, représente une tentative de rétablir un contact entre tous ceux qui se reconnaissent comme élèves directs ou idéels de Brelich. Cet ouvrage ne se veut pas une célébration du maitre (comme prend soin de le préciser Xella dans la préface), mais il n'a certainement pas non plus le ton neutre d'un bilan scientifique sur la pensée de Brelich. Neutre n'a pas été non plus l'ambiance du colloque «Angelo Brelich e la storia delle religioni: problemi e prospettive. Incontro di studio a venticinque anni dalla Sua scomparsa » organisé par le même P. Xella au C.N.R. de Rome (3-4 décembre 2002) pour présenter ce livre. Apparemment, la personnalité de Brelich était tellement fascinante que ceux qui ont eu la chance de l'avoir pour maître ne peuvent en parler de manière neutre. Aux chercheurs plus jeunes ne reste que la nostalgie d'être nés trop tard et de ne pouvoir connaître sa pensée qu'à travers ses écrits. Les articles contenus dans ce recueil représentent un bon point de départ pour rencontrer ce savant, ou un bon moyen pour en approfondir la connaissance.

\section{Francesca Prescendi (Université de Genève)}

Philippe Borgeadd, Aux origines de l'bistoire des religions, Paris, Seuil, 2004. 1 vol. $14 \times 22,5 \mathrm{~cm}, 304$ p. (Libraitie du xxí siècle). ISBN : 2-02-061319-0.

Ce brillant essai est un plaicloyer pour une approche historique, anthropologique et comparatiste de l'histoire des religions. Il intéressera à coup sûr les spécialistes de la religion grecque qui ont à cour de réfléchir sur leur discipline, d'autant que l'A., helléniste lui-même et bien connu notamment pour ses recherches sur le dieu Pan et sur la Mère des dieux, ménage une place de choix au domaine grec, qu'il confronte avec le domaine égyptien et avec le domaine juif, un dernier chapitre scrutant en outre le christianisme dans sa manière d'appréhender le paganisme antique ainsi que les religions païennes du Nouveau Monde. D'une érudition remarquable, ce livre est aussi "engagé », comme on dit; la pensée s'y exprime avec une tranquille assurance, sans sacrifier à la polémique. Une bibliographie copieuse, un index rerum et auctorum (anciens et modernes) ainsi qu'un tableau chronologique (p. 215-217), foumissant des points de repère fort bienvenus, enrichissent le travail. Le report en fin de volume des notes abondantes et parfois très nourries n'est pas fait pour faciliter la lecture, mais l'ouvrage, bien écrit, se lit agréablement et bénéficie d'une présentation soignée; subsistent, comme il est fatal, quelques inadvertances, dont la plus saugrenue est sans doute la date de naissance d'Alexandre $(336:$ p. 216) et la plus plaisante le prénom de Victor dont l'éditeur a affublé la co-auteure, qui n'est pas précisément une inconnue, d'un article sur le bon usage de la notion de syncrétisme (p. 289).

Rendre compte fidèlement de cet ouvrage dans un bref aperçu est une gageure, tant y abondent les sujets traités, les auteurs visités, les questions soulevées, les idées anciennes ou nouvelles qu'on y brasse. Les premières réflexions portent sur l'état actuel des religions, lequel relève bien de l'histoire autant que le passé. Or cet état est celui d'un vaste "bric-à-brac interplanétaire ", un "gigantesque marché aux Puces", où tout n'est en réalité que remploi et bricolage. Il suffit, pour en prendre conscience, d'avoir pratiqué l'histoire comparée des religions, et c'est même là le seul moyen d'y 